\title{
The value of radiographic indexes in the diagnosis of discogenic low back pain: a retrospective analysis of imaging results
}

\author{
Jian Song ${ }^{1, *}$, Hong-Li Wang ${ }^{1, *}$, Xiao-Sheng Ma ${ }^{1, \#}$, Xin-Lei Xia ${ }^{1, *}$, Fei-Zhou Lu ${ }^{1, \#,}$ \\ Chao-Jun Zheng ${ }^{1}$ and Jian-Yuan Jiang ${ }^{1}$ \\ ${ }^{1}$ Department of Orthopaedics, Huashan Hospital, Fudan University, Shanghai, 200040, China \\ *These authors have contributed equally to this work \\ \#Co-authors
}

Correspondence to: Jian-Yuan Jiang, email: jjy@fudanspine.com, jianyuanjiang@sina.com

Keywords: discogenic low back pain, angular motion, modic change, HIZ

Received: March 22, $2017 \quad$ Accepted: May 23, $2017 \quad$ Published: June 27, 2017

Copyright: Song et al. This is an open-access article distributed under the terms of the Creative Commons Attribution License 3.0 (CC BY 3.0), which permits unrestricted use, distribution, and reproduction in any medium, provided the original author and source are credited.

ABSTRACT

To explore value of different radiographic indexes in the diagnosis of discogenic low back pain (LBP). A total number of 120 cases (60 patients diagnosed with discogenic LBP and 60 healthy people) were retrospectively analysed to identify factors in the diagnosis of discogenic LBP by using univariate and multivariate analyses. A receiver operating characteristic (ROC) curve was drew to show the predictive accuracy of the finally enrolled factors. Among all the included patients, 60 were strictly admitted in the discogenic LBP group while the other 60 were enrolled in the control group. Five results shows significant differences between discogenic LBP and control groups, including Cobb angle, lumbar stability, height of the disc, Modic change and High intense zone (HIZ) based on the results of univariate analysis; lumbar stability, Modic change and HIZ show high value in the diagnosis of lumbar discogenic pain based on the multivariate logistic analysis. The ROC curve shows that good diagnostic accuracy was obtained from the enrolled diagnostic factors including Iumbar stability (Angular motion, more than $14.35^{\circ}$ ), Modic change and HIZ.

\section{INTRODUCTION}

LBP has been considered to be the top leading cause for years lived with disability globally, while lumbar discogenic pain is the main cause of LBP [1-3]. Report has confirmed that discogenic LBP is due to degenerative disc disease (DDD) by the magnetic resonance (MR), and the disease can be relieved by the injection of contrast media or local anesthesia into the disc [4-6]. There are a lot of factors which are correlated to the discogenic LBP, such as lumbar spine stability, degree of lumbar intervertebral disc (IVDs) degeneration and pathobiology of Modic changes [7-8]. Recently, studies using animal discogenic pain models and specimens from degenerated human IVDs have provided insights into the pathomechanisms of discogenic LBP, some one believed that painful discs are characterized by a confluence of innervation, inflammation factors [9-10]. Despite its enduring presence, pain ostensibly emanating from a disc itself has hitherto remained poorly defined, and its diagnosis factors have been exceedingly controversial.

Spinal imaging modalities have been widely used to diagnose discogenic LBP, including MR, computed tomography (CT), plain radiography (X-rays), myelography, and CT-myelography, while provocative discographys is the gold standard [11-13]. The most frequent imaging modality was MR, followed by X-rays and CT, while Myelography and CT-myelography alone were scarcely used [5]. However, analysis of imaging measurement in the diagnosis of discogenic LBP still remains absent. Thus, we carried out this research aiming to characterize a carefully selected cohort of the patients with discogenic LBP and to elucidate the factors in the diagnosis of discogenic LBP by using univariate and mulitivariate analyses. 


\section{RESULTS}

\section{Characteristics of enrolled patients}

500 patients diagnosed with LBP were primarily admitted to our study between October 2007 to December 2016 from our hospital. The age of the patients ranged from 30 to 70 years old. We excluded the patients from the studies if they were diagnosed with malignant tumors, spinal tuberculosis, thoracolumbar compression fractures $(n=105)$. A total number of 395 patients were left after this screening. Patients with lumbar disc herniation or lumbar spinal stenosis were also excluded $(n=275) .120$ patients were assessed for eligibility finally. 60 patients with positive results of provocation discography were selected as study group and 60 patients with negative results of provocation discography were regarded as control group (Figure 1). All the characteristics and radiographic results of enrolled patients were specifically listed in the Table 1 .

\section{Results of the univariate analysis}

Univariate analysis was carried out by using student's $t$-test (continuous data) and the $\chi^{2}$ test (dichotomous data). All the results including the basic characteristics and radiographic measurement were fully showed in the Table 1 . The control group owned smaller Cobb angle when compared with the discogenic LBP group $(2.93 \pm 1.91$ vs $3.73 \pm 2.24 ; p=$ 0.04).The patients with discogenic LBP owns lower lumbar stability (Angular motion, lumbar mobility) when compared with the control group $(19.09 \pm 3.44$ vs $10.33 \pm 3.02$, $p<0.001 ; 4.94 \pm 3.18$ vs $2.19 \pm 2.35, p<0.001$ ). The height of the lesion disc in the discogenic LBP group is much lower than the control group (anterior, middle, posterior) (12.68 \pm 1.99 vs $15.67 \pm 2.63, p=0.022 ; 10.88 \pm 2.22$ vs $13.22 \pm$ $1.70, p=0.016 ; 9.38 \pm 2.13$ vs $10.78 \pm 1.29, p=0.028$ ). More Modic changes and HIZ happened in the discogenic LBP group when compared with the control group (55:5 vs $25: 35, p<0.001 ; 31: 29$ vs 7:53 $p<0.001$ ).

\section{Results of multivariate logistic regression}

All statistically significant $(P<0.05)$ covariates based on the univariate analysis, including age of the patients, Cobb angle, lumbar stability, height of the disc, Modic change and HIZ, were subjected to multivariate logistics regression analysis. The results showed that Angular motion, Modic change and HIZ were independently associated with discogenic LBP $(p=0.001$; $p=0.017 ; p=0.013$ ) (Table 2) (Figures 2, 3, 4).

\section{Predictive accuracy of the factors in the diagnosis of discogenic LBP}

A ROC curve was drew to show predictive accuracy of the enrolled factors. The area under the curve of each enrolled factor is more than 0.5 , which were set as reference line, indicating good diagnostic accuracy of the enrolled factors. Angular motion 0.978 (more than $14.35^{\circ}$ ); Modic change 0.747; HIZ 0.717 (Figure 5).

\section{DISCUSSION}

It is not easy to use the symptoms and clinical examinations to diagnose or localize the level of abnormality as the discogenic LBP has a somatotropic rather than dermatomal pattern of pain projection [9]. Several other modalities have been used to help the diagnosis of discogenic LBP, such as MR, CT, myelography, and X-ray [5]. The most frequent imaging modality was MR, which could provides a unique means to evaluate the morphologic status of discs. Study show that MR showed a significant correlation with the discography findings in evaluating disc degeneration as most of the discs with normal morphology on MR were also normal on discography [13]. The clinical relevance of a posterior HIZ on T2-weighted MR images represents fluid or mucoid material associated with granulation tissue and new blood vessels entrapped between torn fibers of the outer annulus or underneath the posterior longitudinal ligament complex [14]. HIZ is mainly due to mechanical fatigue loading, and inflammatory reactions associated with repair of an annular tear. For many patients, the inflammatory mediators such as TNR- $\alpha$, IL-1, IL-6 released following the annular fissures [15-16] may explain the LBP and their high signal intensity on T2-weighted images. According to the results of the univariate analysis, 31 patients $(51.7 \%)$ show posterior HIZ on T2-weighted MR images in the study group while only 7 patients $(11.7 \%)$ in the control group show the same appearance, significant difference was detected between the two groups $(\mathrm{t}=22.1 ; p=0.00)$. We also confirm that high diagnostic value of HIZ were also obtained based on the results of multivariate analysis $(p=0.013)$ and ROC curve(AUC $=0.717)$. So we conclude that HIZ on T2-weighted MR images is credible to forecast the discogenic LBP.

Modic changes appear as the subchondral signal abnormalities on MRI in the bone marrow adjacent to degenerated discs, and it has also be confirmed to be associated with discogenic LBP [17-19]. An recent cadaver research showed that bony endplate lesions had association with the history of LBP [20], and both Modic changes and bony endplate defects could contribute to the LBP. Increased signal intensity (SI) of nucleus pulposus and an accelerated process of disc degeneration have also been confirmed in the process of Modic changes [21-22]. More patients in the symptomatic group own Modic change when compared with the control group (I,17 vs 5; II,31vs $0 ;$ III, 7 vs $0 ; P=0.00$ ) based on the results of the univariate analysis. Modic changes can also applied to forecast the discogenic LBP and show high diagnostic significance according to the results of multivariate analysis $(p=0.017)$ 
Table 1: Characteristics of enrolled discogenic LBP and control group

\begin{tabular}{|c|c|c|c|c|}
\hline Characteristics & Discogenic LBP $(n=60)$ & Control group $(n=60)$ & Value of $\chi^{2}$ or $t$ & $\boldsymbol{P}$ \\
\hline Age of the patients(years) & $50.58 \pm 11.02$ & $50.25 \pm 12.09$ & 0.25 & 0.563 \\
\hline \multicolumn{5}{|l|}{ Gender } \\
\hline Male & $33(55.0 \%)$ & $35(58.4 \%)$ & & \\
\hline Female & $27(45.0 \%)$ & $25(41.6 \%)$ & 0.14 & 0.713 \\
\hline \multicolumn{5}{|l|}{ Disc position } \\
\hline L3-4 & $6(10.0 \%)$ & $8(13.3 \%)$ & 0.32 & 0.570 \\
\hline L4-5 & $37(56.7 \%)$ & $39(65 \%)$ & 0.14 & 0.705 \\
\hline L5-S1 & $17(28.3 \%)$ & $13(21.7 \%)$ & 0.71 & 0.399 \\
\hline Total & & & 0.87 & 0.643 \\
\hline $\begin{array}{l}\text { Cobb angle of Lumbar } \\
\text { vertebra }(\mathrm{T} 12-\mathrm{S} 1)\left({ }^{\circ}\right)\end{array}$ & $3.73 \pm 2.24$ & $2.93 \pm 1.91$ & 2.11 & 0.040 \\
\hline $\begin{array}{l}\text { Lumbar lordosis angle (L1- } \\
\text { S1) }\left(^{\circ}\right)\end{array}$ & $45.3 \pm 8.6$ & $43.6 \pm 7.4$ & 1.20 & 0.100 \\
\hline $\begin{array}{l}\text { Lumbar stability } \text { (lesion } \\
\text { segment) }\end{array}$ & & & & \\
\hline Angular motion $\left({ }^{\circ}\right)$ & $19.09 \pm 3.44$ & $10.33 \pm 3.02$ & 14.85 & 0.000 \\
\hline Lumbar mobility (mm) & $4.94 \pm 3.18$ & $2.19 \pm 2.35$ & 5.35 & 0.000 \\
\hline \multicolumn{5}{|l|}{ Height of the disc $(\mathrm{mm})$} \\
\hline Anterior & $12.68 \pm 1.99$ & $15.67 \pm 2.63$ & -4.96 & 0.022 \\
\hline Middle & $10.88 \pm 2.22$ & $13.22 \pm 1.70$ & 6.19 & 0.016 \\
\hline Posterior & $9.38 \pm 2.13$ & $10.78 \pm 1.29$ & -4.23 & 0.028 \\
\hline \multicolumn{5}{|l|}{$\begin{array}{l}\text { Spinal canal } \\
\text { dimensions(mm) }\end{array}$} \\
\hline Sagittal diameter & $13.98 \pm 2.25$ & $14.09 \pm 3.33$ & -2.01 & 0.054 \\
\hline Tranverse diameter & $23.26 \pm 3.77$ & $24.51 \pm 3.20$ & -1.96 & 0.063 \\
\hline VAS score & $7.6 \pm 2.3$ & $7.2 \pm 1.6$ & 3.2 & 0.143 \\
\hline \multicolumn{5}{|l|}{ Modic change } \\
\hline Negative & $5(8.3 \%)$ & $55(91.7 \%)$ & 33.75 & \\
\hline I & $17(28.3 \%)$ & $5(8.3 \%)$ & 22.34 & \\
\hline II & $31(51.7 \%)$ & $0(0 \%)$ & 51.79 & \\
\hline III & $7(11.7 \%)$ & $0(0 \%)$ & 7.43 & \\
\hline Total & & & 62.02 & 0.000 \\
\hline \multicolumn{5}{|l|}{ HIZ } \\
\hline Positive & $31(51.7 \%)$ & $7(11.7 \%)$ & & \\
\hline Negative & $29(48.3 \%)$ & $53(88.3 \%)$ & 22.18 & 0.000 \\
\hline
\end{tabular}

and ROC curve $(\mathrm{AUC}=0.747)$. The results conducted by our research confirmed the association between the discogenic LBP and Modic change.

$\mathrm{X}$-ray is also widely used to help the diagnosis of discogenic LBP as the angle motion, defined as difference of the flexion and extension angle, can describe degree of the hypermobility. Hypermobility is also thought to play an important role in the IVDs degeneration as IVDs bears major load in humans [23]. Discogenic LBP is mainly due to IVDs degeneration and such process is defined by changes in architecture and biochemical composition that invariably alter the internal mechanical environment of the disc. The anular fibers become torn and disorganized, the nucleus becomes less hydrated and it is difficult to distinguish the border between the anulus and nucleus [10]. Those changes contribute to altering the constraints placed on adjacent vertebrae, leading to spinal hypermobility. Such type of mechanical exposure initiates damage from 
Table 2: Logistic regression of risk factors for discogenic low back pain

\begin{tabular}{lccccc}
\hline \multicolumn{1}{c}{ Factors } & B & S.E. & Wald & P & 95\%CI \\
\hline Angular motion & 0.103 & 0.044 & 5.403 & 0.001 & $0.107 \sim 0.576$ \\
Modic change & -2.688 & 1.129 & 5.673 & 0.017 & $0.007 \sim 0.621$ \\
HIZ & -5.976 & 2.402 & 6.190 & 0.013 & $0.000 \sim 0.281$ \\
Constant & 23.565 & 7.202 & 10.711 & 0.001 & - \\
\hline
\end{tabular}

B: regression coefficient; S.E: standard error

the outside and then works inward via anular delamination and disruption, cellular metaplasia, and vertebral rim hypertrophy as anular fibers is the peripheral disc tissue that acts to restrict intervertebral movement [24]. Ariga et al. [25] have also linked hypermobility to the development of cellular apoptosis and matrix damage within the cartilage end plate by using a mouse mode. The results show that patients in the study group owns much more Angle motion when compared with the patients in the control group $(19.09 \pm 3.44$ vs $10.33 \pm 3.02 ; P=0.00)$, and the it is also the factor showing high diagnostic significance of the lumbar discogenic pain based on the multivariate analysis $(p=0.020)$ and ROC curve $(\mathrm{AUC}=0.978)$.

Discography, regarded as a pain provocation test, has been considered to be the gold standard in the diagnosis of discogenic LBP and it is also the only method that could be applied to relate a radiologic image to the patient's pain directly [26]. The concordant pain at the adjacent disc levels happen in some cases when provocative discographys were carried out for the patients

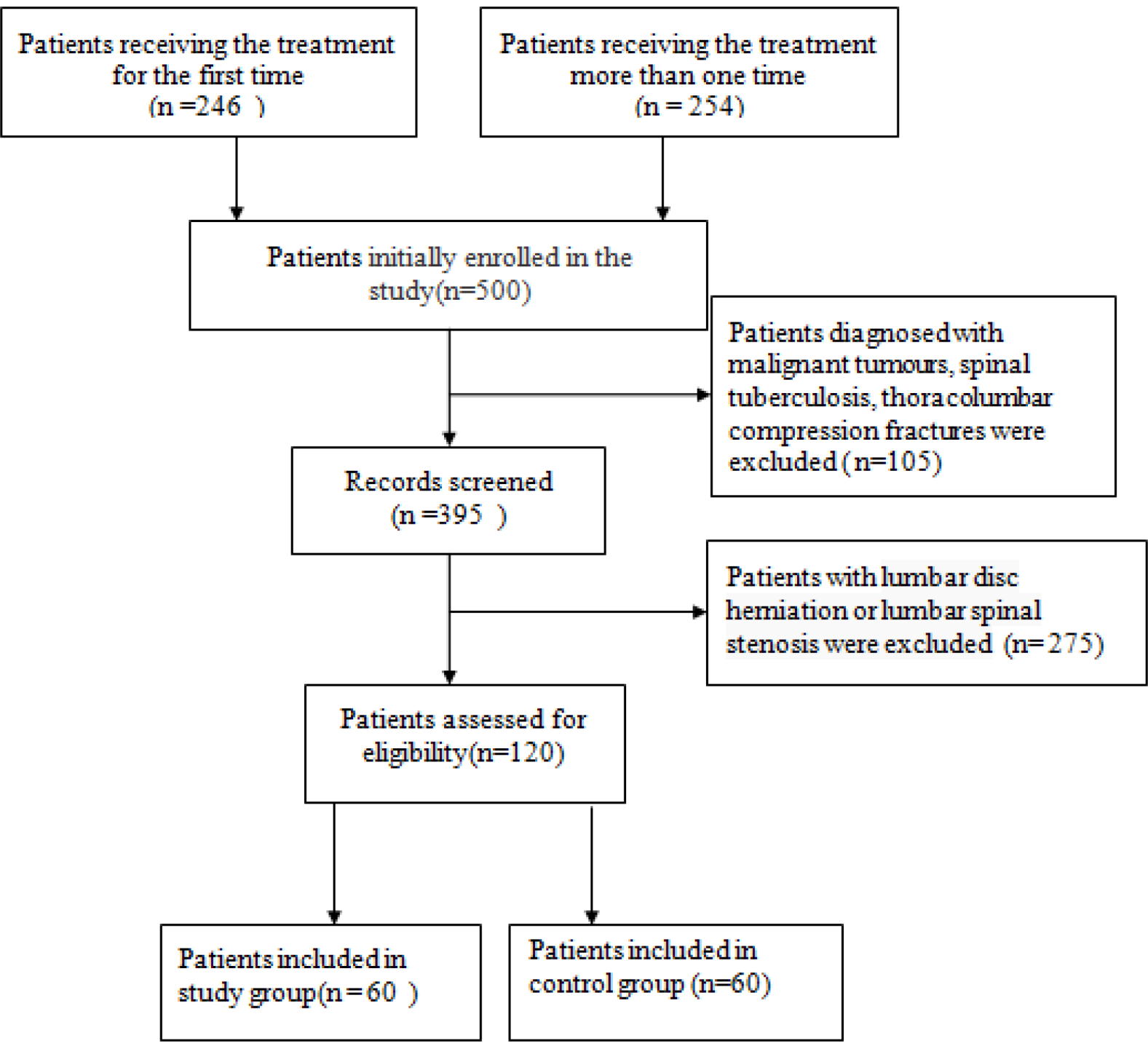

Figure 1: Flow diagram of this population-based study. 

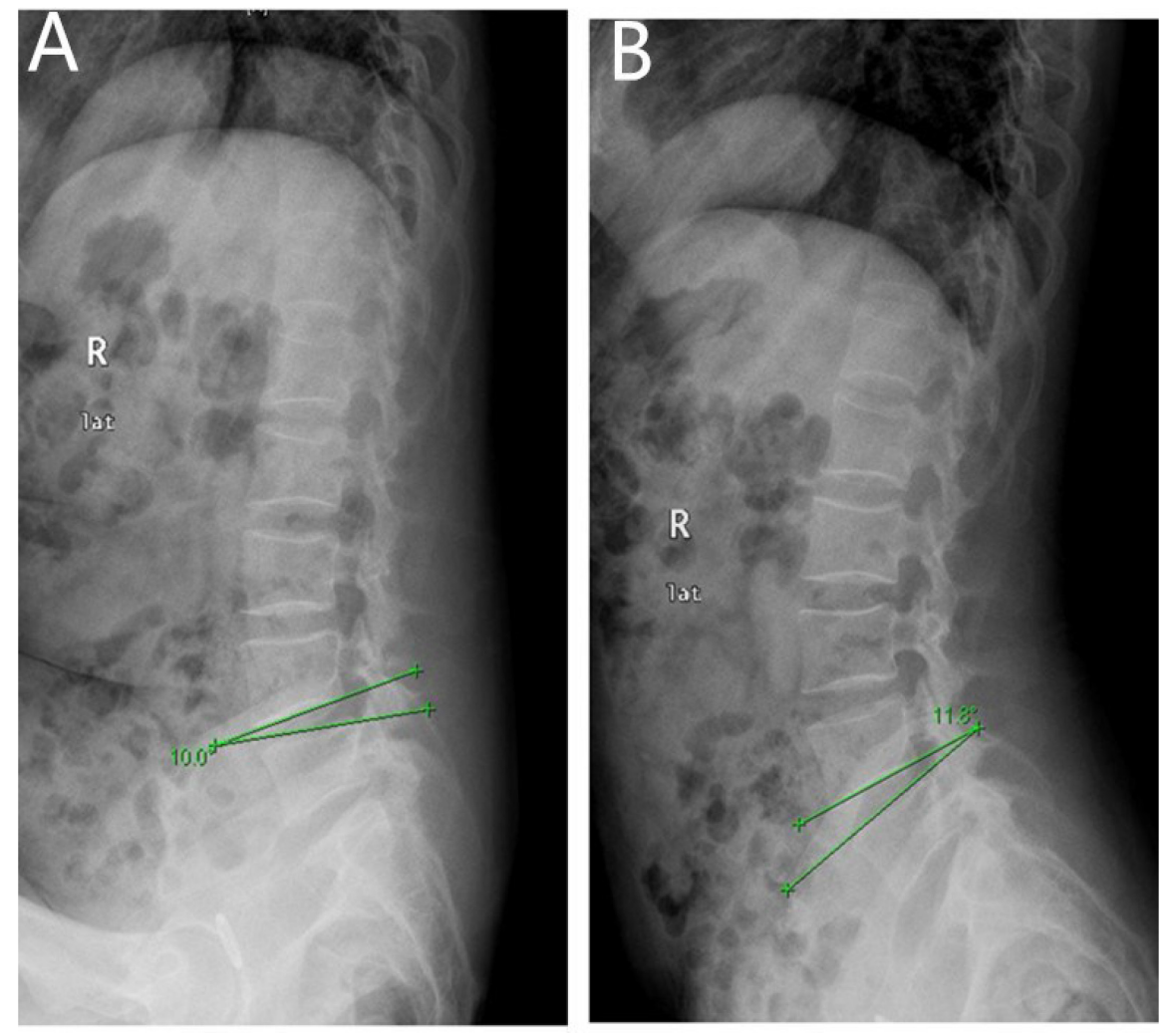

Figure 2: Angular motion of a 48 years old man,diagnosed with discogenic low back pain (LBP) for 2 years. (A) delineates flexion angle measured by X-ray (L4-5). (B) indicates the extension angle measured by X-ray (L4-5).
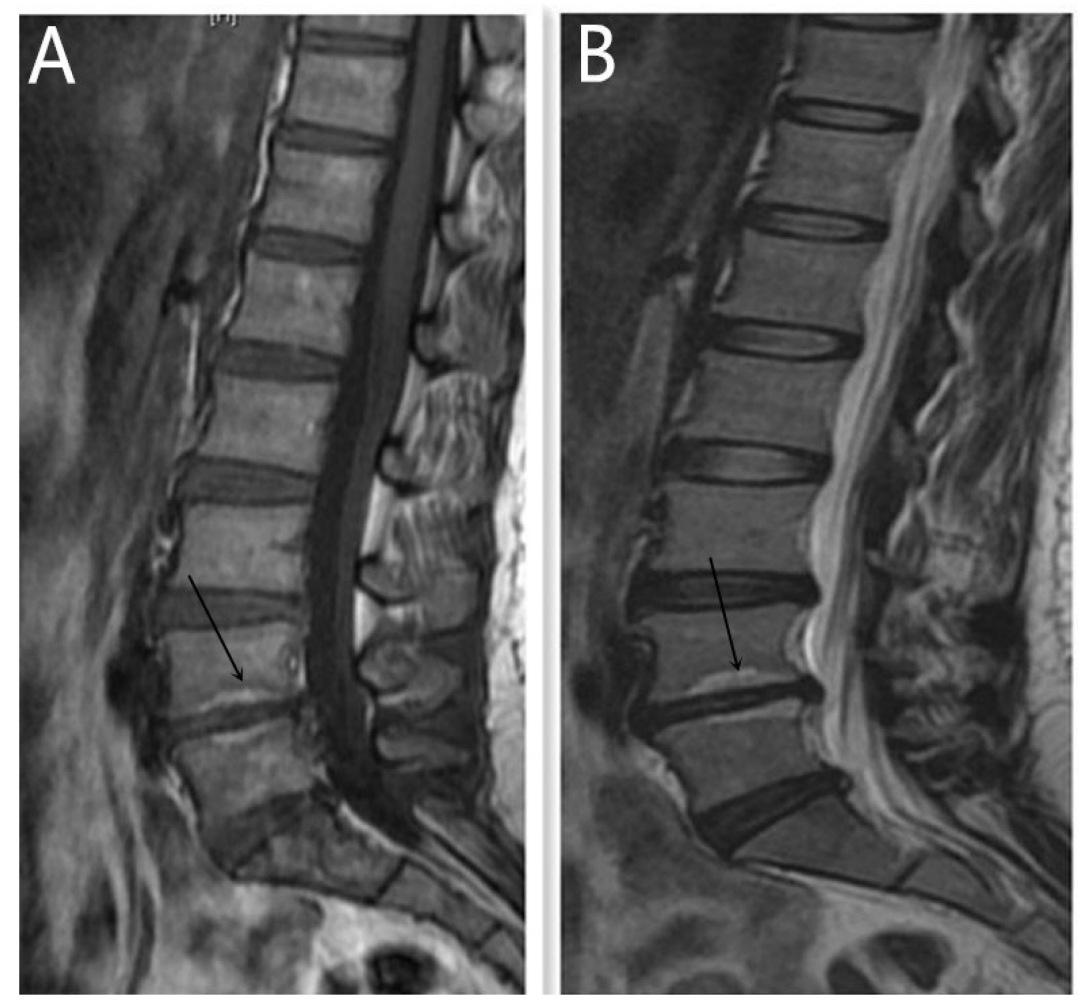

Figure 3: Modic type 2 changes was presented by MR for a 46 year old women, diagnosed with discogenic LBP for 10 months. (A) is High T1 signal (L4-5). (B) indicates High T2 signal (L4-5). 


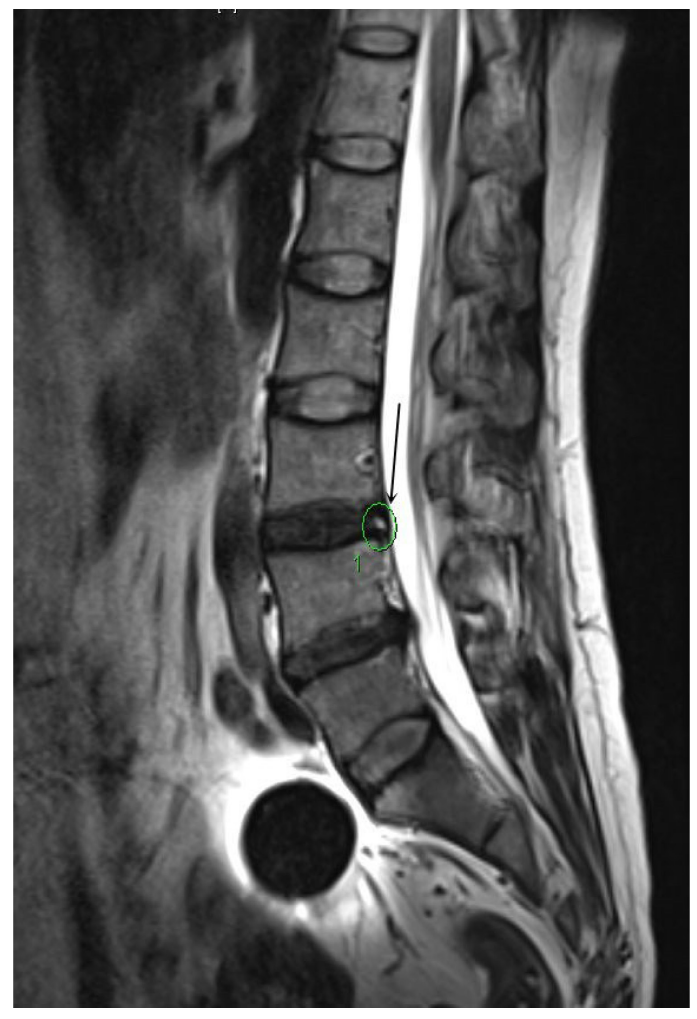

Figure 4: HIZ was presented by MR at the T2 signal (L3-4) for a 56 years old women, diagnosed with discogenic LBP for 3 years.

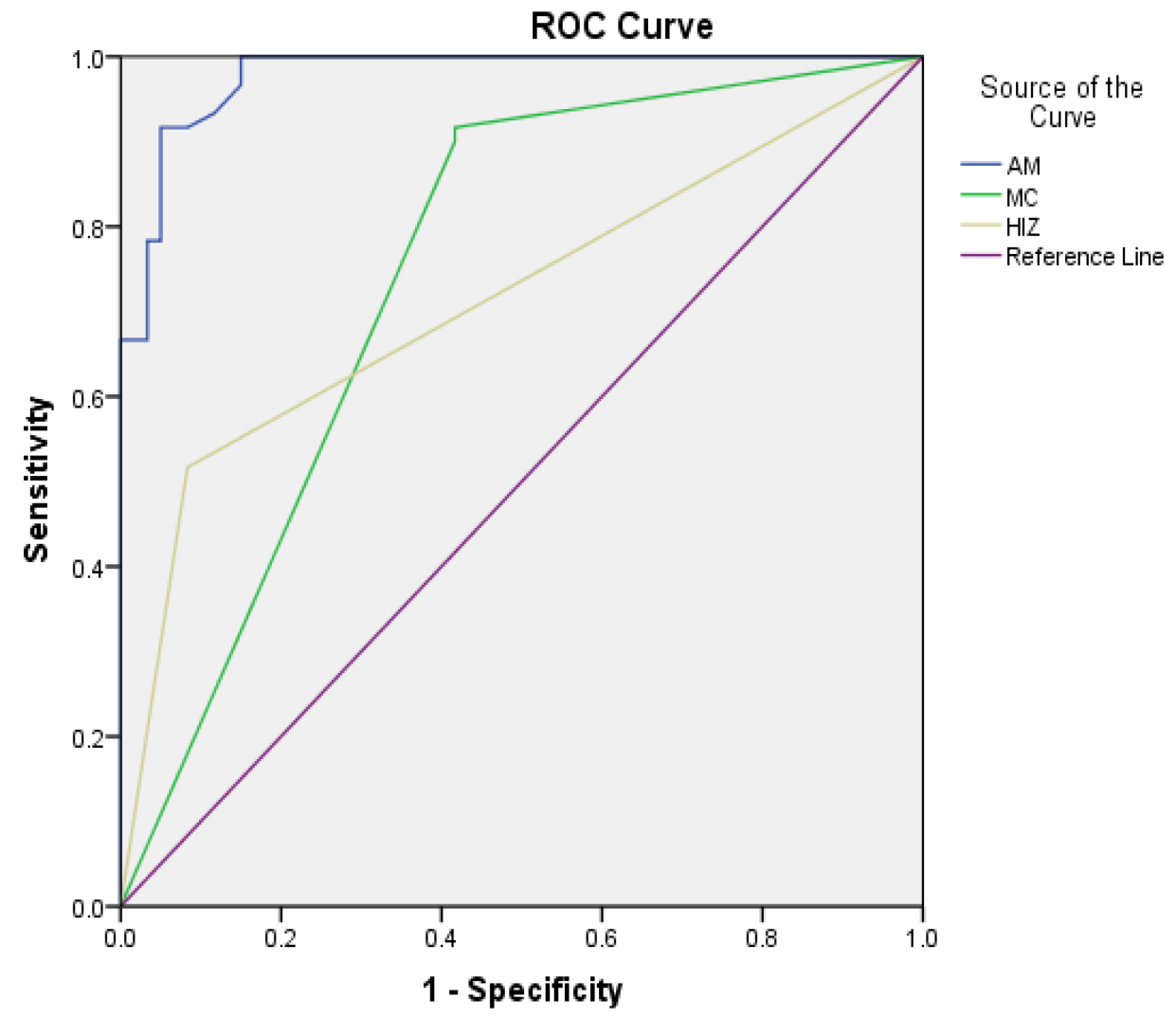

Figure 5: The ROC curve for the diagnosis of discogenic LBP. AM: Angular motion, MC: Modic change, HIZ: high intense zone. 
suspected with discogenic LBP, some were two level lesions, while some others were only one diseased level. According to the diagnostic criteria of discogenic LBP, the lack of concordant pain response at adjacent normal appearing disc levels was required. In order to select those patients with discogenic LBP and position the diseased level correctly, we included those patients with only one lesion level.

Another three results are considered to be associated with discogenic LBP, including age of the patients, Cobb angle, height of the disc; however, they could not be applied to forecast such disease. Discogenic LBP is mainly due to IVDs degeneration, so we hypothesize that it is associated with the increasing age and the result confirmed that based on the univariate analysis [27]. The Cobb angle has become the parameter for quantifying scoliosis curve magnitude. Long term of scoliosis may aggravate local compression and degeneration of the lumbar IVDs. A study has been carried out indicating that LBP was correlated to scoliosis [28]. Research shows that gender (Female), LBP at baseline, radiographic knee osteoarthritis were associated with increased risk for disc height narrowing [29]. IVDs degeneration is associated with progressive morphological, structural, histological, biochemical and functional changes. Severe morphological changes, disc prolapse and end-plate damage, such as anulus tears, are obviously seen with the progression of the disc degeneration. At the stage, disc height narrowing becomes obvious on lumbar radiographs.
Some limitation may exist in the present study. First, this is a retrospective study, as in so many similar published study, may induce section bias. Secondly, the duration of follow-up varied considerably as the enrollment period was so long. Thirdly, the results of univariate and multivariate analyses may be different from the real results because of the limited number of enrolled patients.

\section{MATERIALS AND METHODS}

\section{Ethical considerations}

The study protocol was approved by the ethics committee of Huashan hosital, Fudan university. All the informed consent from the enrolled patients has been acquired as it was a retrospective study. All the methods carried out in the research were performed in accordance with the relevant guidelines and regulations.

\section{Patients enrolling and diagnostic criteria of discogenic LBP}

Patients diagnosed with discogenic LBP were admitted in our study from the department of orthopaedic in our hospital between October 2007 to December 2016; The patients enrolled in the study and control groups should fulfill all of the diagnostic criteria described below: (1) The age of the patients ranged from 30 to 70 years olds. (2) The patients suffered from discontinuous

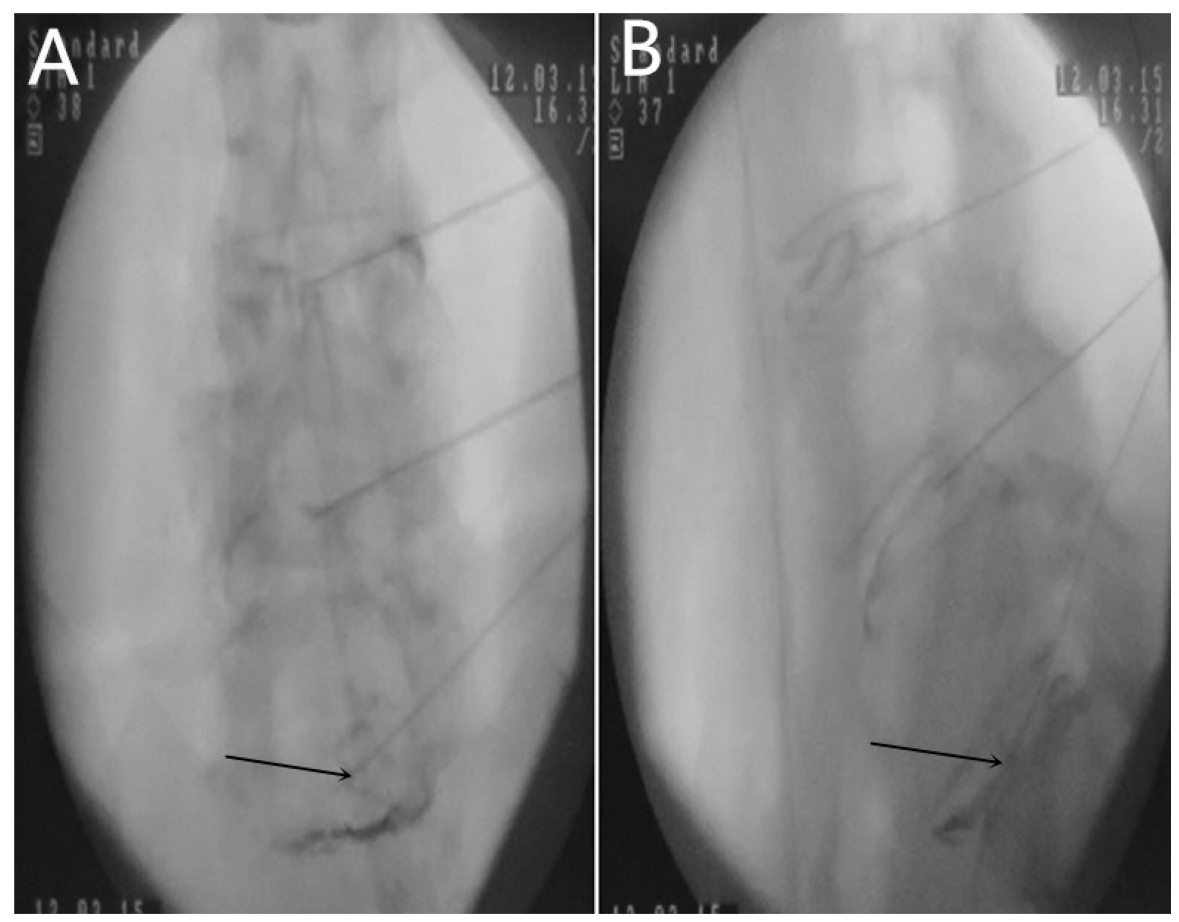

Figure 6: Provocation discography for a 54 years old woman, who had sufferedfrom low back pain (LBP) for more than 2 years. The results shows the ruptured intervertebral disc (L5-S1), leakage of the contrast medium, concordant pain (7 point on the numeric rating scale) was induced according to the VAS score. (A) is the frontal X-ray of the provocation discography. (B) indicates the lateral X-ray of the provocative discography. 
LBP more than 6 months without traumatic history. (3) T2-weighted Magnetic resonance (MR) shows the degenerative intervertebral disc low signal. (4) Concordant pain were recorded during the provocative discography, the presence of a pain response on the Visual Analogue Scale (VAS) of was 6 or higher, adjacent discs show normal appearance or subtle degeneration (one abnormal disc with a normal appearing disc above and below) [30] (5) The level of pfirrman grade is not less than III based on the T2-weighted sagittal images [31]. (6) Only one diseased intervertebral disc is involved (Figure 6). Patients were excluded from the study if they also suffered from malignant tumors, spinal tuberculosis, thoracolumbar compression fractures or some other diseases that could cause LBP (lumbar disc herniation or lumbar spinal stenosis). The same amount of patients with LBP, who own negative results of provocative discography,were enrolled as the control group. For the patients with negative results of provocative discography, two- year follow-up results show that LBP disappears. We also excluded those patients, whose pfirrman grade level was more than III based on the T2-weighted sagittal images in the control group.

\section{Radiographic measurement and analyses of the factors}

Angle motion of the lumbar spine intervertebral was measured to indicate the degree of spine instability and include angle motion and lumbar mobility [32]. Two lines across the upper and lower vertebral body margin of lesion level insect and form the angle at flexion and extension position by X-ray (Phillips,Eindhoven, Netherlands), difference of the flexion and extension angle was defined as the angle motion of lumbar spine [33]. Lumbar mobility was measured by calculating the distance between upper and lower vertebral body of the lesion level at the lateral position by X-ray. The cobb angle of interest is simply the angle between the two line drew from the upper endplate of the upper body and along the lower endplate of the lower body [34]. Height of the lesion disc was measured by supine MR (Siemens,Germany). The T1 and T2 intensity images were constructed with the TE/TR of 10/500 ms and $100 / 2800 \mathrm{~ms}$. The slice thickness was $4 \mathrm{~mm}$. We performed the measurement on the $\mathrm{T} 2$ intensity images at the sagittal planes. The measurement of the vertical intervertebral disc lengths was performed on the midsaggital section of the vertebral body (anterior, middle and posterior). Measurement of spinal canal dimensions of the lesion level (saggital diameter and transverse diameter) was carried out by supine MR to reflect degree of the lumbar spinal stenosis [35]. HIZ was defined as a small, round zone with limited high-intensity signals in the posterior annulus of lumbar intervertebral discs on sagittal slices of T2-weighted MR, it also represents a deep radial fissuring the annulus fibrosis of the lesion level, just as revealed by lumbar CT discography [36-39]. Modic changes are usually displayed by MR to describe the signal intensity changes of vertebral end-plate of the lesion disc [40]. Modic type 1 changes (MC1) refers to low T1 and high T2 signal, MC2 refers to high T1 and $\mathrm{T} 2$ signal, and MC3 refers to low T1 and T2 signal [41]. Grading of disc degeneration was assessed from T2weighted sagittal images based on the Pfirrmann method [31]. Two independent spine surgeons performed the measurement by using Centrieity Enterprise Web V3.0 (General Electric,US).

\section{Statistical analysis}

We used SPSS 21.0 (SPSS, Chicago, IL, USA) to perform all the statistical analyses. Dichotomous data was described as counts and percentages while continuous data are presented as means \pm standard deviation. Univariate analysis was carried out by using student's $t$-test(continuous data) and the $\chi^{2}$ test (dichotomous data). All the performed statistical tests were two-tailed. The statistically significant $(P<0.05)$ covariates based on the univariate analysis were subjected to multivariate logistics regression analysis. A receiver operating characteristic (ROC) curve was drew to show the predictive accuracy while the value of sensibility was set up as vertical coordinate and the value of 1- specificity was set up as the horizontal ordinate. A two-tailed $P$-value $<0.05$ was considered to be statistically significant between the two groups.

\section{CONCLUSIONS}

Lumbar stability (Angular motion, more than $14.35^{\circ}$ ), Modic change and HIZ show high diagnostic value in the diagnosis of discogenic LBP based on the multivariate analysis, good diagnostic accuracy was obtained according to the ROC curve.

\section{Author contributions}

JS, HLW, JYJ conceived the study; JS, WHL, JYJ obtained the samples and carried out the study; JS analyzed data; JYJ provided supervision of the study; JS writed the original draft; HLW, CJZ carried the review and editing. All authors had final approval of the submitted and published versions.

\section{CONFLICTS OF INTEREST}

The authors declared no competing financial interests. 


\section{FUNDING}

This study has received funding by National Natural Science Foundation of China [81472036]; Science and Technology Commission of Shanghai Municipality [16411964100].

\section{REFERENCES}

1. Vos T, Flaxman AD, Naghavi M, Lozano R, Michaud C, Ezzati M, Shibuya K, Salomon JA, Abdalla S, Aboyans V, Abraham J, Ackerman I, Aggarwal R, et al. Years lived with disability (YLDs) for 1160 sequelae of 289 diseases and injuries 1990-2010: a systematic analysis for the Global Burden of Disease Study 2010. Lancet. 2012; 380:2163-2196.

2. Kjaer P, Leboeuf-Yde C, Korsholm L, Sorensen JS, Bendix T. Magnetic resonance imaging and low back pain in adults: a diagnostic imaging study of 40-year-old men and women. Spine (Phila Pa 1976). 2005; 30:1173-1180.

3. Endean A, Palmer KT, Coggon D. Potential of magnetic resonance imaging findings to refine case definition for mechanical low back pain in epidemiological studies: a systematic review. Spine (Phila Pa1976). 2011; 36:160-169.

4. Eck JC, Sharan A, Resnick DK, Watters WR, Ghogawala Z, Dailey AT, Mummaneni PV, Groff MW, Wang JC, Choudhri TF, Dhall SS, Kaiser MG. Guideline update for the performance of fusion procedures for degenerative disease of the lumbar spine. Part 6: discography for patient selection. J Neurosurg Spine. 2014; 21:37-41.

5. Malik KM, Cohen SP, Walega DR, Benzon HT. Diagnostic criteria and treatment of discogenic pain: a systematic review of recent clinical literature. Spine J. 2013; 13:1675-1689.

6. Allegri M, Montella S, Salici F, Valente A, Marchesini M, Compagnone C, Baciarello M, Manferdini ME, Fanelli G. Mechanisms of low back pain: a guide for diagnosis and therapy. F1000Res. 2016; 5 .

7. Dudli S, Fields AJ, Samartzis D, Karppinen J, Lotz JC. Pathobiology of Modic changes. Eur Spine J. 2016; 25:3723-3734.

8. Koenders N, Rushton A, Heneghan N, Verra ML, Willems P, Hoogeboom T, Staal JB. Pain and disability following firsttime lumbar fusion surgery for degenerative disorders: a systematic review protocol. Syst Rev. 2016; 5:72.

9. Ohtori S, Inoue $\mathrm{G}$, Miyagi M, Takahashi $\mathrm{K}$. Pathomechanisms of discogenic low back pain in humans and animal models. Spine J. 2015; 15:1347-1355.

10. Lotz JC, Ulrich JA. Innervation, inflammation, and hypermobility may characterize pathologic disc degeneration: review of animal model data. J Bone Joint Surg Am. 2006; 88:76-82.

11. Ogon I, Takebayashi T, Takashima H, Tanimoto K, Ida K, Yoshimoto M, Fujiwara H, Kubo T, Yamashita T. Analysis of chronic low back pain with magnetic resonance imaging T2 mapping of lumbar intervertebral disc. J Orthop Sci. 2015; 20:295-301.
12. Adams A, Roche O, Mazumder A, Davagnanam I, Mankad K. Imaging of degenerative lumbar intervertebral discs; linking anatomy, pathology and imaging. Postgrad Med J. 2014; 90:511-519.

13. Devine J G. Commentary: Standardization of dynamic lumbar imaging and diagnostic criteria for discogenic low back pain. Spine J. 2011; 11:999-1001.

14. Peng B, Hou S, Wu W, Zhang C, Yang Y. The pathogenesis and clinical significance of a high-intensity zone (HIZ) of lumbar intervertebral disc on MR imaging in the patient with discogenic low back pain. Eur Spine J. 2006; 15: 583-587.

15. Burke JG, Watson RW, McCormack D, Dowling FE, Walsh MG, Fitzpatrick JM. Intervertebral discs which cause low back pain secrete high levels of proinflammatory mediators. J Bone Joint Surg Br. 2002; 84:196-201.

16. Iwasawa $\mathrm{T}$, Iwasaki $\mathrm{K}$, Sawada $\mathrm{T}$, Okada A, Ueyama K, Motomura S, Harata S, Inoue I, Toh S, Furukawa KI. Pathophysiological role of endothelin in ectopic ossification of human spinal ligaments induced by mechanical stress. Calcif Tissue Int. 2006; 79:422-430.

17. Jensen TS, Karppinen J, Sorensen JS, Niinimäki J, LeboeufYde C. Vertebral endplate signal changes (Modic change): a systematic literature review of prevalence and association with non-specific low back pain. Eur Spine J. 2008; 17:1407-1422.

18. Maatta JH, Wadge S, MacGregor A, Karppinen J, Williams FM. ISSLS Prize Winner: Vertebral Endplate (Modic) Change is an Independent Risk Factor for Episodes of Severe and Disabling Low Back Pain. Spine (Phila Pa 1976). 2015; 40:1187-1193.

19. Mok FP, Samartzis D, Karppinen J, Fong DY, Luk KD, Cheung KM. Modic changes of the lumbar spine: prevalence, risk factors, and association with disc degeneration and low back pain in a large-scale populationbased cohort. Spine J. 2016; 16:32-41.

20. Wang Y, Videman T, Battie MC. ISSLS prize winner: Lumbar vertebral endplate lesions: associations with disc degeneration and back pain history. Spine (Phila Pa 1976). 2012; 37:1490-1496.

21. Luoma K, Vehmas T, Gronblad M, Kerttula L, Kaapa E. Relationship of Modic type 1 change with disc degeneration: a prospective MRI study. Skeletal Radiol. 2009; 38:237-244.

22. Kerttula L, Luoma K, Vehmas T, Gronblad M, Kaapa E. Modic type I change may predict rapid progressive, deforming disc degeneration: a prospective 1-year followup study. Eur Spine J. 2012; 21:1135-1142.

23. Nachemson AL, Schultz AB, Berkson MH. Mechanical properties of human lumbar spine motion segments. Influence of age, sex, disc level, and degeneration. Spine (Phila Pa 1976). 1979; 4:1-8.

24. Wada E, Ebara S, Saito S, Ono K. Experimental spondylosis in the rabbit spine. Overuse could accelerate the spondylosis. Spine (Phila Pa 1976). 1992; 17:S1-S6.

25. Ariga K, Miyamoto S, Nakase T, Okuda S, Meng W, Yonenobu K, Yoshikawa H. The relationship between 
apoptosis of endplate chondrocytes and aging and degeneration of the intervertebral disc. Spine (Phila Pa 1976). 2001; 26:2414-2420.

26. Guyer RD, Ohnmeiss DD. Lumbar discography. Position statement from the North American Spine Society Diagnostic and Therapeutic Committee. Spine (Phila Pa 1976). 1995; 20:2048-2059.

27. Gautschi OP, Smoll NR, Joswig H, Corniola MV, Schaller K, Hildebrandt G, Stienen MN. Influence of age on pain intensity, functional impairment and health-related quality of life before and after surgery for lumbar degenerative disc disease. Clin Neurol Neurosurg. 2016; 150:33-39.

28. Balague F, Pellise F. Adolescent idiopathic scoliosis and back pain. Scoliosis Spinal Disord. 2016; 11:27.

29. Akeda K, Yamada T, Inoue N, Nishimura A, Sudo A. Risk factors for lumbar intervertebral disc height narrowing: a population-based longitudinal study in the elderly. BMC Musculoskelet Disord. 2015; 16:344.

30. Knop C, Oeser M, Bastian L, Lange U, Zdichavsky M, Blauth M. Development and validation of the Visual Analogue Scale (VAS) Spine Score. [Article in German]. Unfallchirurg. 2001; 104:488-497.

31. Pfirrmann CW, Metzdorf A, Zanetti M, Hodler J, Boos N. Magnetic resonance classification of lumbar intervertebral disc degeneration. Spine (Phila Pa 1976). 2001; 26:1873-1878.

32. Harvey S, Hukins D, Smith F, Wardlaw D, Kader D. Measurement of lumbar spine intervertebral motion in the sagittal plane using videofluoroscopy. J Back Musculoskelet Rehabil. 2016; 29:445-457.

33. Dupuis PR, Yong-Hing K, Cassidy JD, Kirkaldy-Willis WH. Radiologic diagnosis of degenerative lumbar spinal instability. Spine (Phila Pa 1976). 1985; 10:262-276.
34. Morrissy RT, Goldsmith GS, Hall EC, Kehl D, Cowie GH. Measurement of the Cobb angle on radiographs of patients who have scoliosis. Evaluation of intrinsic error. J Bone Joint Surg Am. 1990; 72:320-327.

35. Schenck C, van Susante J, van Gorp M, Belder R, Vleggeert-Lankamp C. Lumbar spinal canal dimensions measured intraoperatively after decompression are not properly rendered on early postoperative MRI. Acta Neurochir (Wien). 2016; 158:981-988.

36. Stern D, Likar B, Pernus F, Vrtovec T. Parametric modelling and segmentation of vertebral bodies in 3D CT, MR spine images. Phys Med Biol. 2011; 56:7505-7522.

37. Aprill C, Bogduk N. High-intensity zone: a diagnostic sign of painful lumbar disc on magnetic resonance imaging. Br J Radiol. 1992; 65:361-369.

38. Khan I, Hargunani R, Saifuddin A. The lumbar highintensity zone: 20 years on. Clin Radiol. 2014; 69:551-558.

39. Shan Z, Chen H, Liu J, Ren H, Zhang X, Zhao F. Does the high-intensity zone (HIZ) of lumbar Intervertebral discs always represent an annular fissure? Eur Radiol. 2016; 27:1267-1276.

40. Luoma K, Vehmas T, Kerttula L, Gronblad M, Rinne E. Chronic low back pain in relation to Modic changes, bony endplate lesions, and disc degeneration in a prospective MRI study. Eur Spine J. 2016; 25:2873-2881.

41. Laustsen AF, Bech-Azeddine R. Do Modic changes have an impact on clinical outcome in lumbar spine surgery? A systematic literature review. Eur Spine J. 2016; 25:3735-3745. 